\title{
Competitive binding of musclin to natriuretic peptide receptor 3 with atrial natriuretic peptide
}

\author{
Shunbun Kita ${ }^{1}$, Hitoshi Nishizawa ${ }^{2}$, Yosuke Okuno ${ }^{2}$, Masaki Tanaka ${ }^{3}$, Atsutaka Yasui ${ }^{1,3}$, \\ Morihiro Matsuda ${ }^{2}$, Yukio Yamada ${ }^{1}$ and lichiro Shimomura ${ }^{2,3}$ \\ ${ }^{1}$ Pharmaceutical Research Division, Pharmacology Research Laboratories I, Takeda Pharmaceutical Company Ltd, Yodogawa-ku, Osaka 532-8686, Japan \\ ${ }^{2}$ Department of Metabolic Medicine, Graduate School of Medicine and ${ }^{3}$ Department of Medicine and Pathophysiology, Graduate School of Frontier Bioscience, \\ Osaka University, 2-2 Yamadaoka, Suita, Osaka 565-0871, Japan \\ (Correspondence should be addressed to I Shimomura; Email: ichi@imed2.med.osaka-u.ac.jp; S Kita; Email: kita_shunbun@takeda.co.jp)
}

\begin{abstract}
Musclin is a novel skeletal muscle-derived secretory factor that was isolated by our group. Musclin contains a region homologous to natriuretic peptides (NPs). This study investigated the interaction between musclin and NP receptors (NPRs). Musclin specifically bound to NPR3, but not to NPR1 or NPR2. Musclin and atrial natriuretic peptide (ANP) competed for binding to NPR3. We conducted binding assays using various synthetic musclin peptides and mutant musclin proteins. The first NP-homologous region in musclin $\left({ }^{88} \mathrm{LDRL}^{91}\right)$ and the second
\end{abstract}

homologous region $\left({ }^{117} \mathrm{MDRI}^{120}\right)$ were responsible cooperatively for high-affinity binding to NPR3. The first NP-homologous region was more importantly associated with binding to NPR3, than the second homologous region. The competitive nature of musclin with ANP for the natriuretic clearance receptor NPR3 was also confirmed in vivo. We conclude that musclin binds to NPR3 competitively with ANP and may affect ANP concentrations in a local or systemic manner.

Journal of Endocrinology (2009) 201, 287-295

\section{Introduction}

Skeletal muscle is a major energy-expending organ important for regulating glucose, lipid, and protein metabolism. It was demonstrated recently that this tissue produces and secretes biologically active molecules, conceptualized as myokines (Musarò et al. 2001, Nishizawa et al. 2004, Salih et al. 2004, Sell et al. 2006). We and others recently identified one of these novel secretory factors, and named musclin/osteocrine respectively (Thomas et al. 2003, Nishizawa et al. 2004). We demonstrated that musclin mRNA expression was almost exclusive in the skeletal muscle of rodents, and induced in obesity models. We also showed that musclin mRNA expression decreased during fasting and increased upon refeeding (Nishizawa et al. 2004, Yasui et al. 2007).

The musclin protein contains a region homologous to members of the natriuretic peptide (NP) family, as well as a KKKR putative serine protease cleavage site, also characteristic of NP proteins (Nishizawa et al. 2004). These findings suggested that musclin signaling could be related to that of NP family and its receptors. The mammalian NP family comprises the atrial natriuretic peptide (ANP), B-type natriuretic peptide (BNP), and C-type natriuretic peptide (CNP; Potter et al. 2006). These proteins function as endocrine/paracrine factors in regulating blood pressure and extracellular fluid volume, fat metabolism, and skeletal development (Potter et al. 2006). To date, three singlemembrane-spanning NP receptors (NPRs) have been identified. NPR 1 and NPR2 are guanylyl cyclase receptors that regulate cGMP levels, while NPR3 lacks enzymatic activity and may act as a clearance receptor (Potter et al. 2006).

The present study investigated interactions between musclin and NPRs, with a special focus on the competition between musclin with ANP for the natriuretic clearance receptor NPR3.

\section{Experimental procedures}

\section{Plasmids}

Full-length wild-type musclin (WT (30-130); 30-130 amino acid excluding N-terminal signal sequence) or mutant (mutant (80-130); 80-130 amino acid) mouse musclin cDNA was inserted into the ligation independent cloning (LIC)-cloning site of pET32Xa-LIC (Merck KGaA) to produce Escherichia coli-derived recombinant proteins. PCRbased point mutations were introduced at amino acid residues Asp89Gly and Arg90Gly to encode mutations in ANPhomologous region 1 (Mutant-1), and at Asp118Gly to encode a mutation in ANP-homologous region 2 (Mutant-2) in full-length (30-130 amino acid) mouse musclin cloned in 
pET32Xa-LIC. All three mutations were introduced to encode mutations in both ANP-homologous regions 1 and 2 (Mutant-3). The corresponding mutations in C-terminal half musclin (cWT; mutant (80-130)) were introduced in Mutant-1C (Asp89Gly and Arg90Gly), Mutant-2C (Asp118Gly), and Mutant-3C (all three mutations) respectively.

Full-length (30-130 amino acids) mouse musclin cDNA was cloned into pET15b (Merck KGaA). The residue located in the thrombin cleavage site between the N-terminal His x6 tag and the musclin cDNA was mutated from Leu-Val-ProArg-Gly-Ser to Leu-Val-Pro-Tyr-Gly-Cys (His x6 YC-musclin). The Tyr and Cys are necessary for N-terminal tyrosyliodination and maleimide biotinylation respectively. The N-terminal iodinated one is designated as $\left[{ }^{125} \mathrm{I}\right]-$ Musclin, and the $\mathrm{N}$-terminal biotinylated one through the Cys residue is designated as BC-Musclin.

Full-length (30-130 amino acid) mouse musclin cDNA was cloned into the pFLAG-CMV3 mammalian expression vector (Sigma-Aldrich, Inc.) to encode N-terminal FLAGtagged mouse musclin (FLAG-WT).

The mammalian expression vectors of human NPR Npr1/pCMVXL6, Npr2/pCMVXL4, and Npr3/pCM VXL4 were purchased from OriGene Technologies Inc. (Rockville, MD, USA). The extracellular domain of mouse NPR 3 cDNA was cloned from the first strand cDNA of lung prepared from normal $\mathrm{C} 57 \mathrm{Bl} / 6 \mathrm{~J}$ mice. The extracellular regions of human (aa1-481) and mouse (aa1-477) NPR3 were tandem-inserted with the human IgG-Fc coding sequence (aa240-471 of human IgG1) into pcDNA3.1 (-) for producing $\mathrm{Fc}$ fusion constructs (hNPR3-IgGFC and mNPR3-IgGFc respectively; Bennett et al. 1991).

\section{Recombinant proteins}

Recombinant GST-fusion proteins were produced using E. coli Rosseta gami B cells carrying the pET32Xa-LIC musclin fusion vector, and were purified with $\mathrm{Ni}$-agarose. The resultant fusion proteins were cleaved by factor $\mathrm{Xa}$, purified using HiTrap SP cation exchange resin (GE Healthcare Biosciences, Uppsala, Sweden) equilibrated with $25 \mathrm{mM}$. HEPES, pH 7.9, and eluted with increasing concentration of $\mathrm{NaCl}(0 \cdot 1-1 \mathrm{M})$ in the equilibration buffer. The full-length musclin protein (WT (30-130)) was biotinylated with an eightfold molar excess of sulfo-NHSLC-biotin (Pierce, Rockford, IL, USA), and designated as BK-Musclin. The efficacy of biotin incorporation into the musclin molecule was calculated by the HABA method, with 4.8 biotin molecules incorporated into one molecule of musclin. His x6 YC-musclin protein was produced by E. coli Rosseta gami B cells carrying the modified pET15b-musclin expression plasmid constructed as above, and purified with Ni-agarose and HiTrap SP cation exchange resin. The purified protein was iodinated by lactoperoxidase with $\mathrm{Na}^{125}$ I to produce ${ }^{125}$ I-labeled musclin ([ $\left.{ }^{125} \mathrm{I}\right]-$ Musclin), or was biotinylated with maleimide-PEO2 biotin (Pierce;
BC-Musclin), followed by TCEP disulfide reduction according to the instructions provided by the manufacture. The efficacy of biotin incorporation was almost 1 biotin per 1 musclin molecule. Partial peptides of 105-130, and 80-112 amino acids of musclin were synthesized by CosmoBio Co. (Tokyo, Japan).

FreeStyle293 cells (Invitrogen) were cultured according to the instructions provided by the manufacturer. The N-terminal FLAG-WT was produced by transfecting FreeStyle293 cells with the pFLAG-CMV3 vector containing fulllength mouse musclin cDNA, according to the instructions provided by the manufacturer. Three days after transfection, the culture medium was collected and centrifuged at $440 \mathrm{~g}$ for $15 \mathrm{~min}$ at $4{ }^{\circ} \mathrm{C}$. The resultant supernatant was adjusted to $\mathrm{pH}$ $7 \cdot 5$ with $\mathrm{NaOH}$, and bound to HiTrap SP cation exchange resin (GE Healthcare Biosciences), washed with $25 \mathrm{mM}$ HEPES, pH 7.5 and $50 \mathrm{mM} \mathrm{NaCl}$, and then eluted with $25 \mathrm{mM}$ HEPES, pH 7.5 and $1000 \mathrm{mM} \mathrm{NaCl}$. The eluate was then applied to anti-FLAG agarose conjugate (SigmaAldrich) pre-equilibrated with the equilibration buffer containing $50 \mathrm{mM}$ Tris- $\mathrm{HCl}, \mathrm{pH} 7 \cdot 4,150 \mathrm{mM} \mathrm{NaCl}$, one complete protease inhibitor tablet/100 ml buffer (Roche Diagnostics), and $0 \cdot 1 \%$ Tween 20 . Following several washes with the equilibration buffer, the desired protein was eluted with $100 \mu \mathrm{g} / \mathrm{ml}$ flag peptide included in the same buffer. The peak fraction monitored at $280 \mathrm{~nm}$ was collected and desalted using a PD-10 column (GE Healthcare) pre-equilibrated with sterile PBS.

ANP (rat and mouse), ANP (104-126) Des-[Cys105, Cys121] (L-ANP, rat and mouse), ANP (4-23)-Amide Des-[Gln18, Ser19, Gly20, Leu21, Gly22] (C-ANP, rat and mouse) and biotin-labeled ANP (rat and mouse) were purchased from Phoenix Pharmaceuticals Inc. Burlingame, CA, USA. [ $\left.{ }^{125} \mathrm{I}-\mathrm{Tyr} 28\right]$ ANP, rat $\left(\left[{ }^{125} \mathrm{I}\right]-\mathrm{ANP}\right.$, IM186) was purchased from GE Healthcare Inc. (Potter et al. 2006).

The Fc fusion constructs were transfected into FreeStyle293 mammalian expression cells (Invitrogen) according to the instructions provided by the manufacturer. $F_{C}$ fusion proteins were purified as described (Bennett et al. 1991), with minor modifications. After 4 days, the cell supernatants were pooled and purified with HiTrap rProtein A column equilibrated with PBS containing $0 \cdot 1 \% \mathrm{NaN}_{3}$, and eluted with Gentle Ag/Ab elution buffer, pH 6.9 (Pierce 21027). The peak elution was dialyzed against PBS and concentrated with 10000 molecular cut-off ultrafiltration membrane (YM-10, Millipore, Bedford, MA, USA).

\section{Binding assay}

BW5147 cells stably expressing NPR 3 were generated using retroviral transduction with pMXs-IG-NPR3. BW5147NPR 3 cells $\left(2 \times 10^{5}\right)$ were incubated at $4{ }^{\circ} \mathrm{C}$ for $30 \mathrm{~min}$ with binding buffer $(0 \cdot 1 \% \mathrm{BSA} / \mathrm{HANKS}$ ' balanced salt solution) containing $1 \mathrm{nM}$ biotinylated full-length musclin (BKMusclin) or $1 \mu \mathrm{M}$ biotinylated ANP. For competition experiments, BW5147-NPR 3 cells $\left(2 \times 10^{5}\right)$ were incubated 
at $4{ }^{\circ} \mathrm{C}$ for $30 \mathrm{~min}$ with binding buffer containing $10 \mathrm{nM}$ biotinylated-musclin (BK-Musclin) with varying concentrations of non-biotinylated recombinant full-length musclin (WT (30-130)), ANP (rat and mouse), insulin or adiponectin. The cells were then washed twice with binding buffer, and incubated at $4{ }^{\circ} \mathrm{C}$ for $30 \mathrm{~min}$ with binding buffer containing streptavidin-allophycocyanin (GE Healthcare Biosciences). The cells were washed twice, and the fluorescence intensity of the samples was measured using a FACS Calibur instrument (BD Biosciences).

FreeStyle293 cells $\left(1 \times 10^{6}\right)$ transiently expressing human NPR 1, NPR 2 or NPR 3 were incubated on ice for $60 \mathrm{~min}$ with $10 \mathrm{nM}$ of ${ }^{125}$ I-labeled full-length musclin $\left(\left[{ }^{125} \mathrm{I}\right]-\right.$ Musclin) prepared above or $0 \cdot 1 \mathrm{nM}\left[{ }^{125} \mathrm{I}\right]-\mathrm{ANP}$, in the absence (control) or presence of competitors indicated in the figure legends. The cells were then separated by passing through a dibutylphthalate:dinonylphthalate (1:1) layer, and tubes were frozen in liquid nitrogen. The radioactivity associated with cells in the bottom of the tube was counted using a $\gamma$-counter. The radioactivity associated with cells in the absence of competitors (control) was taken as $100 \%$ in each cell expressing NPR1, NPR2 or NPR3 respectively.

For the binding studies using IgG-Fc fusion NPR3, black immunoplates were coated at $4{ }^{\circ} \mathrm{C}$ overnight with $1.0 \mu \mathrm{g} / \mathrm{ml}$ anti-human IgG-Fc affinity-purified monoclonal antibody (A80-105A, Bethyl Laboratories, Montgomery, TX, USA) dissolved in $100 \mu \mathrm{l}$ of $0 \cdot 1 \mathrm{M} \mathrm{NaCO}_{3}$ and $0 \cdot 1 \mathrm{M} \mathrm{NaCl}(\mathrm{pH}$ $8 \cdot 3)$. The plates were then blocked with Tris-buffered saline (TBS; $20 \mathrm{mM}$ Tris, $150 \mathrm{mM} \mathrm{NaCl}$, and $0 \cdot 1 \% \mathrm{NaN}_{3}$ ) containing 25\% (V/V) ImmunoBlock reagent (DS Pharma Biomedical Co., Osaka, Japan) at room temperature for $2 \mathrm{~h}$. The mouse or human NPR3-IgGFc-mediated capture binding assay was originally developed and optimized as follows. The optimized reaction mixture contained $0.3 \mu \mathrm{g} / \mathrm{ml}$ human NPR3-IgGFc protein or $3.0 \mathrm{ng} / \mathrm{ml}$ mouse NPR3-IgGFc protein, $0 \cdot 1 \mathrm{ng} / \mathrm{ml} \mathrm{N}$-terminal biotinylated full-length musclin (BC-Musclin) protein, in the absence (control) or presence of the indicated concentrations of unlabeled competitors as described in the figure legends, in a total of $100 \mu \mathrm{l}$ TBS containing $5 \%(\mathrm{~V} / \mathrm{V})$ ImmunoBlock reagent, and $0 \cdot 1 \%$ Tween 20 . The binding reaction was performed at room temperature for $16 \mathrm{~h}$. Following two washes with $250 \mu \mathrm{l}$ TBS containing $0 \cdot 1 \%$ Tween 20, streptavidin-conjugated $\beta$-galactosidase (Roche, 1112481) diluted to $1 / 10000$ with the same buffer was incubated at room temperature for $1 \mathrm{~h}$. After four further washes with $250 \mu \mathrm{l}$ TBS containing $0 \cdot 1 \%$ Tween 20 , the plates were incubated at room temperature for $3 \mathrm{~h}$ with buffer containing $10 \mathrm{mM} \mathrm{K}_{2} \mathrm{HPO}_{4}, 150 \mathrm{mM} \mathrm{NaCl}, 2 \mathrm{mM} \mathrm{MgCl} 2,0 \cdot 1 \% \mathrm{BSA}$, $0 \cdot 1 \% \mathrm{NaN}_{3}$, and $0.5 \mathrm{mM} 4$-Methylumbelliferyl $\beta$-D-galactopyranoside, $\mathrm{pH} 7 \cdot 0$. Following the addition of $150 \mu \mathrm{l} 0 \cdot 1 \mathrm{M}$ glycine solution, $\mathrm{pH} 10 \cdot 3$, fluorescent intensities were measured at an excitation wavelength of $355 \mathrm{~nm}$ and emission wavelength of $460 \mathrm{~nm}$. The fluorescence developed in the absence of any competitors was taken as the control value, and the fluorescence developed without $\mathrm{N}$-terminal biotinylated full-length musclin (BC-Musclin) protein was taken as the baseline. For the calculation of percent inhibition, the following equation was used; $100-100 \times(($ fluorescence in the presence of competitor) - (baseline fluorescence) $) /($ control fluorescence) - (baseline fluorescence)).

\section{Animal studies}

Male C57BL/6J mice (CLEA Japan, Tokyo, Japan), between 10 and 13 weeks old, were anesthetized with pentobarbital sodium $\left(65 \mathrm{mg} / \mathrm{kg}\right.$ i.p.) and their body was heated to $40{ }^{\circ} \mathrm{C}$ for $30 \mathrm{~min}$. The right jugular vein was then catheterized, and $45 \mu \mathrm{l}$ blood was collected from the tail vein and placed in iced tubes containing EDTA and aprotinin. Immediately, saline, C-ANP (4-23; Bachem, Bubendorf, Switzerland) or recombinant musclin from $E$. coli was administered as a bolus injection $(100 \mu \mathrm{g} / \mathrm{kg}$ body weight) followed by constant infusion (10 $\mu \mathrm{g} / \mathrm{min}$ per kg body weight) for $1 \mathrm{~h}$, after which a further $45 \mu \mathrm{l}$ blood was collected from the tail vein. Plasma samples were stored at $-80{ }^{\circ} \mathrm{C}$ until RIA for ANP. All experimental protocols were approved by the Ethics Review Committee for Animal Experimentation of Osaka University.

\section{Measurement of plasma $A N P$}

Plasma was assayed for ANP levels using the $\alpha$-rat ANF RIA kit (Bachem) according to the protocol supplied by the manufacturer. C-ANP (4-23) at concentrations up to $10 \mu \mathrm{g} / \mathrm{ml}$ was completely devoid of cross-reactivity with the antibody.

\section{Statistical analysis}

Data were expressed as mean \pm s.D., except for experiments in vivo (expressed as means \pm s.E.M. in Fig. 5). Unpaired Student's $t$-test was used to evaluate differences between two groups, unless otherwise specified. A $P$ value $<0 \cdot 05$ denoted a statistically significant difference. IC $_{50}$ values were calculated by probit analysis, and expressed with 95\% confidence interval in the figure legends.

\section{Results}

We previously identified musclin as a novel secretory factor isolated from mouse skeletal muscle (Nishizawa et al. 2004). The musclin amino acid sequence is highly conserved among mouse, rat, and human, with an N-terminal signal sequence and two C-terminal regions highly homologous with ANP (Nishizawa et al. 2004). Furthermore, musclin contains putative serine protease cleavage sites proximally upstream of the ANP-homologous regions (Fig. 1). However, in contrast to ANP, musclin has no conserved cysteine residue by which ANP forms a ring structure to bind its receptor, NPR1. Based on these structural differences, we hypothesized that musclin might bind specifically to the natriuretic 

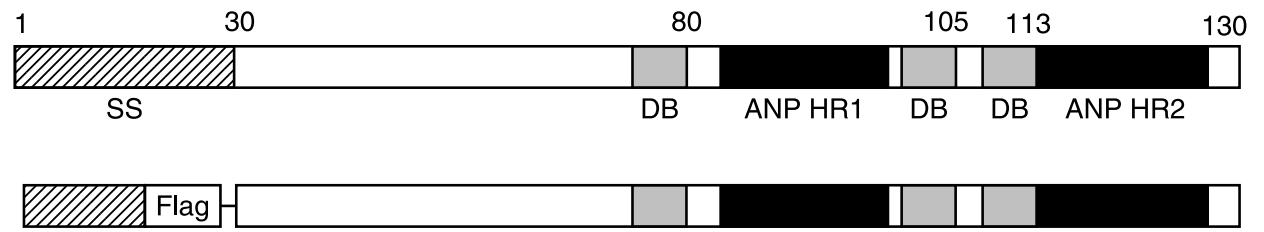

Ig leader
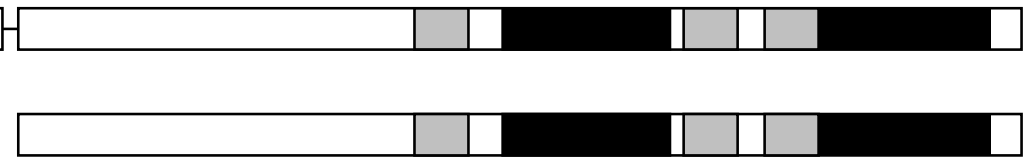

His $x 6-Y C$

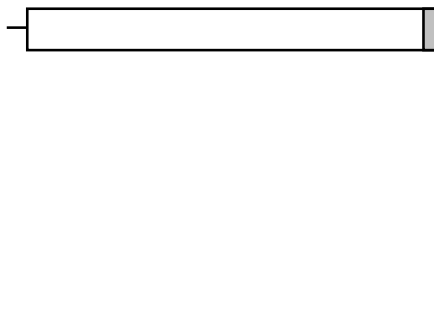

Mouse

musclin

full length

$(1-130)$

Flag-musclin

WT (30-130) and BK-Musclin

His $x 6$ YC-musclin and BC-Musclin

cWT (80-130)

$80-112$

$105-130$

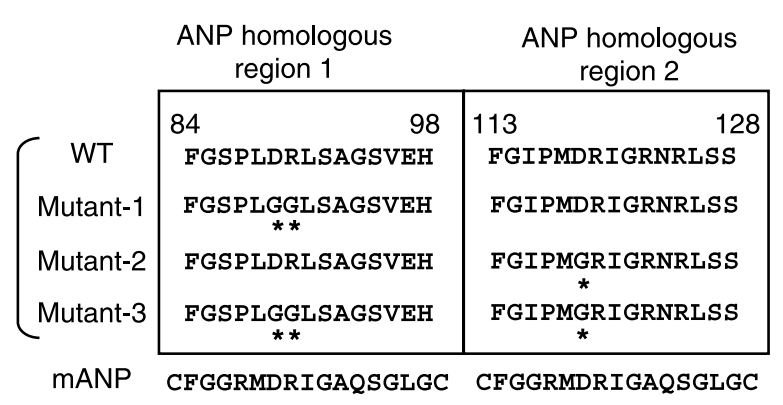

Figure 1 Schematic presentation of the deletion peptides of musclin used in this study. The full-length and deletion peptides of musclin used throughout this study are summarized in schematic presentation (not to scale). Putative full-length musclin (WT (30-130)) lacking possible signal sequence (1-29 amino acids) and C-terminal half musclin (cWT (80-130)) corresponding to a possible cleavage product at the first dibasic region were prepared as E. coli recombinant proteins. Partial peptides of 105-130 and 80-112 amino acids of musclin were chemically synthesized. WT (30-130) was labeled with biotin sulfo-NHS-LC-biotin to incorporate biotin molecules into native lysine residue and was designated as BK-Musclin. N-terminal His x6-tagged putative mature musclin (30-130 amino acids) produced in E.coli. was labeled with ${ }^{125} \mathrm{I}$ or maleimide-PEO2 biotin to produce [ $\left.{ }^{125} \mathrm{I}\right]-\mathrm{Musclin}$ or N-terminal-biotinylated full-length musclin (BC-Musclin). SS, possible signal sequence; DB, dibasic peptides; ANP, atrial natriuretic peptide; $H R$, homologous region.

clearance receptor NPR3, particularly given that a mutant ANP lacking the ring structure can also bind NPR3 (Maack et al. 1987, Potter et al. 2006). The full-length and deletion peptides of musclin used throughout this study are summarized in schematic presentation (Fig. 1).

To elucidate whether musclin can specifically bind to any NPR among the NPR family, $\left[{ }^{125} \mathrm{I}\right]$-labeled mouse musclin $\left(\left[{ }^{125} \mathrm{I}\right]\right.$-Musclin; N-terminal His x6 tagged WT (30-130)) binding to FreeStyle293 cells transiently expressing human NPR1, 2 or 3 was examined (Fig. 2a). Specific binding of $\left[{ }^{125} \mathrm{I}\right]-$ Musclin that comprises the binding that could be inhibited with excess amount of non-labeled musclin was observed only with cells expressing hNPR 3, and not with those expressing hNPR1 or hNPR2 (Fig. 2a). By contrast, $\left[{ }^{125} \mathrm{I}\right]$-labeled ANP bound specifically to each of the NPRtransfected cells (Fig. 2b). Similar to the case of C-ANP and L-ANP, which are known to bind specifically to NPR3, musclin slightly inhibited ANP binding to NPR1, did not inhibit ANP binding to NPR2 at all, and specifically inhibited ANP binding to NPR3 (Fig. 2b). Specific binding of $\left.{ }^{125} \mathrm{I}\right]-$ Musclin or $\left[{ }^{125} \mathrm{I}\right]-$ labeled ANP was negligibly-small in non-transfected cells (data not shown). These results clearly indicated that musclin binds to NPR3 in a competitive fashion with ANP.

To elucidate the binding affinity of musclin to NPR3 more precisely, the binding of biotinylated recombinant mouse musclin (BK-Musclin; WT (30-130) biotinylated at Lys residues) to BW5147 cells stably expressing mouse NPR3 was evaluated by FACS analysis. The fluorescent intensity of mNPR 3 expressing cells increased with the amount of biotinylated musclin added to the reaction (Fig. 3a), and a negligibly-small increase was observed in parent BW5147 cells not expressing mNPR3 (data not shown), suggesting that musclin could bind to NPR3 expressed on the cell surface. Next, unlabeled musclin (WT (30-130)) or ANP were added into the reaction to examine the competitive nature of the 

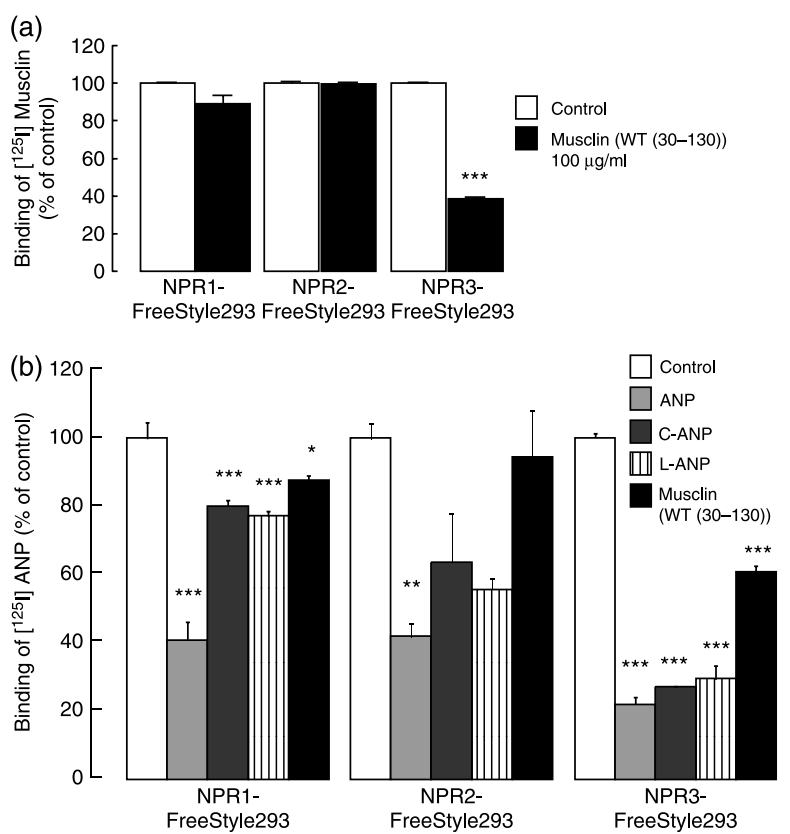

Figure 2 Specific binding of musclin to NPR3. (a) FreeStyle293 cells transiently expressing hNpr1/pCMV6-XL6, hNpr2 or hNpr3/pCMV6-XL4 were incubated with $10 \mathrm{nM}\left[{ }^{125} \mathrm{I}\right]$-labeled musclin ([ $\left.{ }^{125} \mathrm{I}\right]-$ Musclin) in the absence (Control) or presence of $100 \mu \mathrm{g} / \mathrm{ml}$ full-length musclin (WT (30-130)). The radioactivity associated with cells in the absence of competitors (controls) was taken as $100 \%$ in each cells expressing NPR1, NPR2 or NPR3 respectively. The specific binding that corresponds to the difference of $\left[{ }^{125} \mathrm{I}\right]$-Musclin binding to control cells minus in the presence of excess non-labeled musclin (WT (30-130)) was observed only in the cells expressing hNpr3. (b) FreeStyle293 cells transiently expressing hNpr1/pCMV6-XL6, hNpr2 or hNpr3/pCMV6-XL4 were incubated with $0 \cdot 1 \mathrm{nM}\left[{ }^{125} \mathrm{I}\right]-\mathrm{ANP}$, in the absence (control) or presence of $100 \mu \mathrm{g} / \mathrm{ml}$ ANP, circular ANP (C-ANP), linear ANP (L-ANP), and WT (30-130) of musclin. The radioactivity associated with cells in the absence of competitors (controls) was taken as $100 \%$ in each cells expressing NPR1, NPR2 or NPR3 respectively. The specific bindings that correspond to the difference of [ $\left.{ }^{125} \mathrm{I}\right]-\mathrm{ANP}$ binding to control cells minus in the presence of excess non-labeled ANP was observed in any cells expressing hNpr1, 2 or 3, but not observed in non-transfected cells (data not shown). [ $\left.{ }^{125} \mathrm{I}\right]$-ligands associated with cells were separated by passing through the dibutylphthalate:dinonylphthalate $(1: 1)$ layer, and counted using a $\gamma$ counter. Data are mean \pm S.D. $(n=3-4) .{ }^{*} P<0 \cdot 01 .{ }^{*} P<0 \cdot 001$. ${ }_{* * *} P<0 \cdot 0001$. Similar results were obtained in another independent experiment.

musclin/NPR3 interaction. Musclin, $8 \cdot 8$-fold more strongly than ANP, competitively displaced the binding between biotinylated musclin and the NPR3-expressing cells (Fig. 3b). By contrast, structurally different secreting factors, insulin, and adiponectin, had no such effect (Fig. 3b).

Next, the musclin region responsible for the NPR3 binding was investigated by the binding of $\mathrm{N}$-terminal biotinylated musclin (BC-Musclin; N-terminal His x6 tagged WT (30-130) biotinylated at the N-terminal added Cys residue. Refer to Fig. 1) to an $\mathrm{IgG}-\mathrm{Fc}$ fusion protein of human NPR3 (hNPR3-IgGFc), and then adding partial peptides of musclin (Fig. 4a). Musclin binding to NPR3 was also confirmed in these binding experiments using hNPR3-IgGFc, since recombinant musclin (WT (30-130), closed circles) and ANP (gray circles) competed with the N-terminal biotinylated musclin (BC-Musclin) binding to hNPR3-IgGFc (Fig. 4a). The cWT (80-130), closed triangles), which corresponds to the region downstream of the first putative dibasic cleavage site ${ }^{76} \mathrm{KKKR}^{79}$, inhibited musclin binding similar to WT (30-130; closed circles). Next, we investigated which part of ANP-homologous regions downstream of ${ }^{76} \mathrm{KKKR}^{79}$ is more important for musclin binding to NPR3, with two overlapping synthetic peptides encompassing 105-130 (open triangles) and 80-112 (open squares). Peptide 105-130 (open triangles) showed no obvious binding inhibition, but peptide 80-112 (open squares) significantly inhibited the binding in a concentration-dependent manner (Fig. 4a). These results suggested that the first ANP-homologous region of musclin from ${ }^{76} \mathrm{KKKR}^{79}$ to ${ }^{110} \mathrm{KKR}^{113}$ is more important for binding to NPR3. In addition, and it is noteworthy that the mouse full-length musclin expressed in mammalian cells (Flag-WT, closed squares) inhibited this binding 3-4-fold more strongly than the E. coli-derived, recombinant full-length musclin (WT (30-130), closed circles) or 2·4-fold than ANP (gray circles; Fig. 4a), which suggests that native musclin may have more potent binding affinity to NPR3, than ANP.

To elucidate the contribution of two ANP-homologous regions more precisely, we next constructed mutants of musclin with amino acid changes in two ANP-homologous regions (Fig. 1). The competitive binding of mutants with full-length musclin (WT (30-130), closed circles) for the mNPR 3-IgGFc fusion protein was examined (Fig. 4b). Mutant-1 (closed squares) has two amino acid changes at Asp89Gly and at Arg90Gly in ANP-homologous region 1 (aa94-98), while Mutant-2 (open circles) has a single amino acid change at Asp118Gly in ANP-homologous region 2 (aa113-128). Prior to this experiment, we established that mouse musclin bound more strongly to mouse NPR3 (mNPR3-IgGFc) than to human NPR3 (hNPR3-IgGFc) in the IgG-Fc fusion system (data not shown), and used mNPR3-IgGFc in this experiment. Although musclin (WT (30-130), closed circles) potently inhibited N-terminal biotinylated musclin binding with $0 \cdot 2 \mathrm{nM}$ of $\mathrm{IC}_{50}$ value, Mutant-1 (closed squares) and Mutant-2 (open circles) exhibited more than 75 -fold and $4 \cdot 7$-fold reduction in inhibitory potency as compared with full-length musclin (WT (30-130)). These observations shown in Fig. 4b indicated that the mutation in ANP-homologous region 1 reduced the binding affinity of musclin for NPR 3 10-fold more than those in ANP-homologous region 2. Mutant-3 (open squares) that has both mutations in ANP-homologous regions 1 and 2 virtually abolished the binding to NPR3. The result suggested that NP-homologous region 1 and 2 are cooperatively responsible for high-affinity binding to NPR3. 

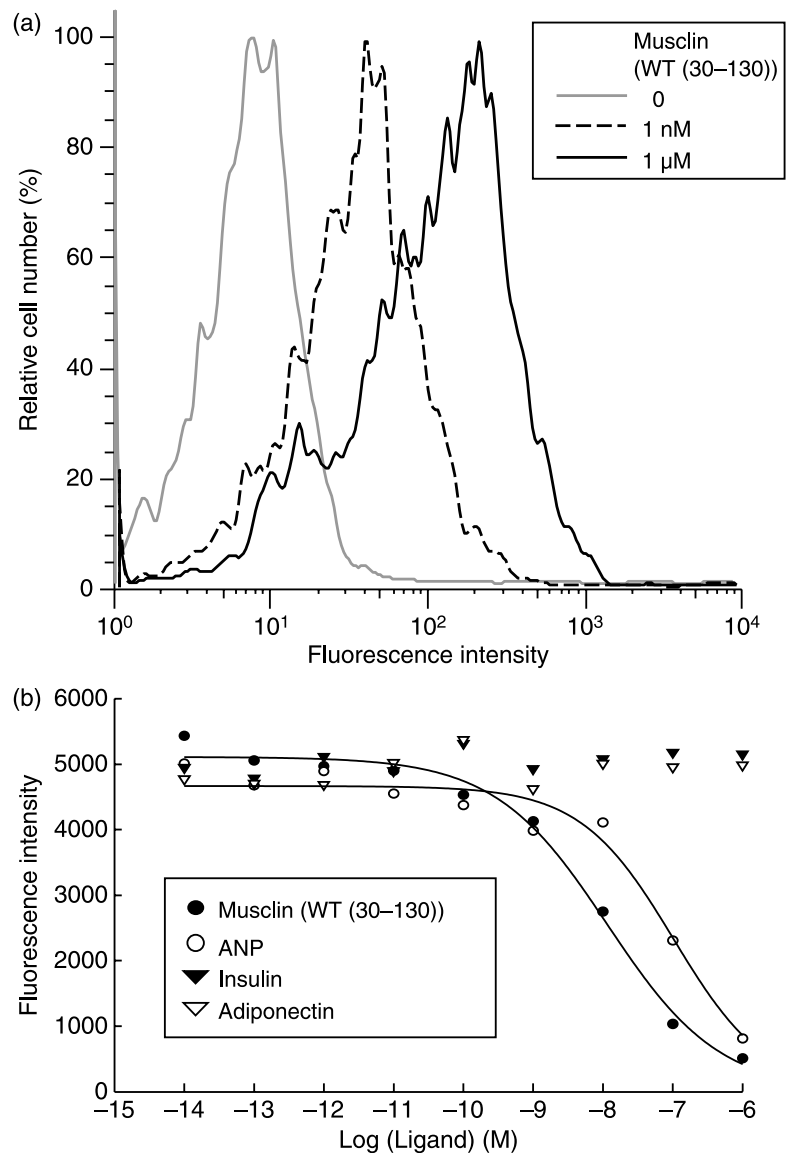

Figure 3 Binding of musclin to cellular NPR3. (a) BW5147-mNPR3 cells stably expressing mNPR3 were incubated without (gray lines) or with $1 \mathrm{nM}$ (broken lines) and $1 \mu \mathrm{M}$ (solid lines) of biotinylated full-length musclin (BK-Musclin). Fluorescence intensity (FL4) was analyzed by FACS. Percent of relative cell numbers, top as $100 \%$, were represented against fluorescent intensity. (b) BW5147-mNPR3 cells were incubated with $10 \mathrm{nM}$ BK-Musclin and varying concentrations of non-biotinylated ligands, including full-length musclin (WT (30-130); closed circles), ANP (open circles), insulin (closed triangles), and adiponectin (open triangles). Mean fluorescence intensity at indicated concentrations of non-biotinylated ligand was analyzed by FACS. IC 50 value with $(95 \%$ confidence interval) by full-length musclin (WT (30-130)) was $11.5 \mathrm{nM}(6 \cdot 3-$ $19 \cdot 7 \mathrm{nM})$, and by ANP was $102 \mathrm{nM}(38.9-224 \mathrm{nM})$ respectively. Similar results were obtained in three independent experiments.

These reduced binding affinities of musclin to NPR3-IgGFc were recapitulated by introducing the same mutations in cWT (80-130; open circles), which corresponds to cWTMutant-1 (closed squares, 200-fold), cWT-Mutant-2 (open circles, 3-fold) and cWT-Mutant-3 (open squares, diminished); Fig. 4c).

Finally, because NPR 3 is a clearance receptor for NPs, we investigated the effects of musclin on plasma endogenous ANP in C57BL/6J mice (Fig. 5). Maack et al. (1987) reported that constant infusion of C-ANP, a synthetic NPR3 ligand, increased plasma ANP by decreasing the clearance rate of
ANP (Almeida et al. 1989). As expected, constant infusion of recombinant full-length musclin (WT (30-130)) in mice significantly increased plasma ANP to a degree similar to that observed with C-ANP (Fig. 5). These results suggest that infused musclin competed for the binding of circulating ANP for NPR3 in vivo as well.

\section{Discussion}

The present study demonstrated several features of musclin binding to NPR3: 1) musclin selectively binds to NPR 3 with high affinity among the NPR family; 2) musclin binds to NPR3 with higher affinity than ANP; 3) the first of two ANP-homologous regions in the cWT is important for binding to NPR3; 4) mammalian-expressed musclin shows higher affinity binding to NPR 3 than E. coli-derived musclin; and, 5) systemic infusion of recombinant musclin increases plasma ANP concentrations, possibly via competing for the natriuretic clearance receptor NPR3.

We originally identified musclin as a novel secretory factor from skeletal muscle (Nishizawa et al. 2004). The present study demonstrated that musclin specifically and selectively binds to NPR3, among the NPR family. As described previously, the NP family member most homologous to musclin, ANP, binds to all of the NPR family members, NPR1, NPR2, and NPR3. Consistent with these findings, only the ANP/NPR 3 binding was competed for by musclin, with similar strength to C-ANP and L-ANP, two ligands specific for NPR3. L-ANP is artificially linearized, resulting in no Cys-Cys loop structure used by ANP to bind NPR1. NPR 3 itself has no guanylyl cyclase domain required for signal transduction, and was thus proposed to function as a clearance receptor for the NP molecules (Yasui et al. 2007). Musclin has no cysteine residues, suggesting that it could not form a disulfide-bonded intermolecular loop structure, as in ANP. It also has three dibasic putative cleavage sites. Collectively, these data suggest that musclin exists as a linear peptide, with potential only to bind NPR3.

The specific binding of musclin was similarly observed on stably NPR3-transfected BW5147 cells and transiently NPR3-transfected HEK293 cells, and not observed on NPR1- nor NPR2-transfected HEK293 cells. Furthermore, musclin inhibited labeled musclin binding more strongly than ANP, which has been considered to be the highest affinity ligand of NPR 3 among NPs. Binding was observed using an IgGFc-fusion protein, independent of labeling methods. Musclin binding to NPR3 was inhibited by unlabeled recombinant full-length musclin produced in E. coli and by ANP, in NPR 3 expressed on the cell surface or fused with IgG-Fc. The N-terminal Flag-WT expressed in mammalian cells inhibited musclin binding to NPR 3 more strongly than E. coli-produced musclin and ANP. This strong inhibition of mammalian-expressed musclin binding was also observed on cells expressing NPR3 (data not shown). The musclin protein expressed in mammalian cells has a molecular size of $20 \mathrm{kDa}$, 

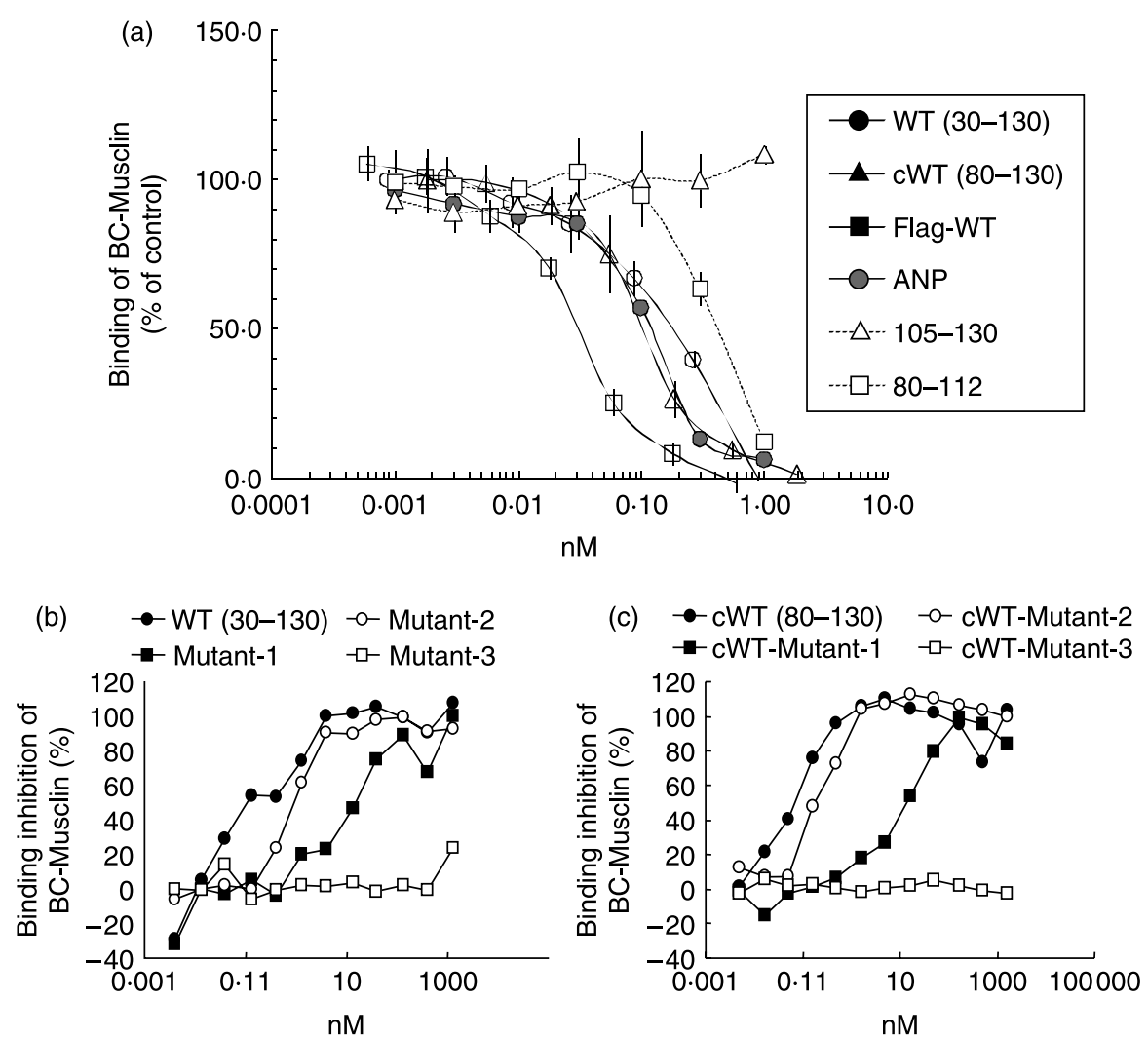

Figure 4 Characterization of musclin binding to NPR3. (a) E. coli-derived full-length musclin (WT (30-130)), C-terminal half musclin (cWT (80-130)), mammalian-derived N-terminal FLAGtagged full-length musclin (Flag-WT), ANP (atrial natriuretic peptide), and peptides corresponding to aa105-130, and 80-112 of musclin were incubated with N-terminal-biotinylated full-length musclin (BC-Musclin) and IgG-Fc fusion human NPR3 (hNPR3-IgGFc) in anti-IgG-Fc antibody precoated plates. The fluorescent intensity associated with bound BC-Musclin, which was developed with streptavidin- $\beta$-galactosidase were measured. The results are expressed as a percentage of the control (without non-labeled competitor), taken as 100\%, and baseline (without IgG-Fc fusion human NPR3) taken as $0 \%$. Data are mean \pm s.D. $(n=3)$. $I C_{50}$ value with $(95 \%$ confidence interval) by each peptide was as follows, WT (30-130); 0.14 nM (0.12-0.19 nM), cWT (80-130); 0.10 nM $(0 \cdot 088-0 \cdot 12 \mathrm{nM})$, Flag-WT; 0.044 nM (0.035-0.056 nM), ANP; 0.11 nM (0.088-0.13 nM), and aa80-112; 0.39 nM (0.34-0.45 nM). (b) Full-length musclin (WT (30-130)), Mutant-1, Mutant-2, and Mutant-3 or (c) C-terminal half musclin (cWT (80-130)), cWT-Mutant-1, cWT-Mutant-2, and cWT-Mutant-3, which have corresponding mutations in C-terminal half musclin (cWT (80-130)), as described in detail in Fig. 1, were incubated with $0.1 \mathrm{ng} / \mathrm{ml} \mathrm{N}$-terminal-biotinylated full-length musclin (BC-Musclin) and $3 \mathrm{ng} / \mathrm{ml} \operatorname{lgG}-F c$ fusion mouse NPR3 (mNPR3-IgGFc). The fluorescent intensity associated with bound BC-Musclin was measured and the results are expressed as a percentage inhibition of the control (without non-labeled competitor), taken as $0 \%$ inhibition, and baseline (without mNPR3-IgGFc) taken as $100 \%$ inhibition. Data are average values of $n=2 . \mathrm{IC}_{50}$ value with (95\% confidence interval) by each peptide was as follows, WT (30-130); $0 \cdot 20 \mathrm{nM}$ $(0 \cdot 066-0 \cdot 60 \mathrm{nM})$, Mutant-1; $15 \cdot 2 \mathrm{nM}(4 \cdot 6-50 \mathrm{nM})$, Mutant-2; 0.95 nM (0.72-1.2 nM), cWT (80-130); 0.062 nM (0.033-0.12 nM), cWT-Mutant-1; $13 \mathrm{nM}(7 \cdot 7-20 \mathrm{nM})$, and cWT-Mutant-2; $0 \cdot 19 \mathrm{nM}(0 \cdot 12-0 \cdot 31 \mathrm{nM})$. Essentially same result was obtained in another independent experiment.

which is larger than the $11 \mathrm{kDa}$ predicted from the amino acids sequence (Nishizawa et al. 2004). Taken together, these data indicate that the higher-order structure of musclin, including the sugar-chain modifications, is important for the binding to NPR3.

In the binding studies using NPR3-IgGFc fusion protein, the cWT mutant showed the same or more potent inhibitory activity than full-length protein. Peptides corresponding to aa105-130 showed lower inhibitory activities than aa80-114, suggesting that the first of the two ANP-homologous regions in musclin has a more significant role in the binding to NPR3. In addition, we could confirm the relative importance of the first ANP-homologous region using mouse NPR3IgGFc, which binds with higher affinity to mouse musclin. The two consecutive amino acid mutations of 89DG to $90 \mathrm{RG}$ in the first ANP-homologous region (Mutant-1) 


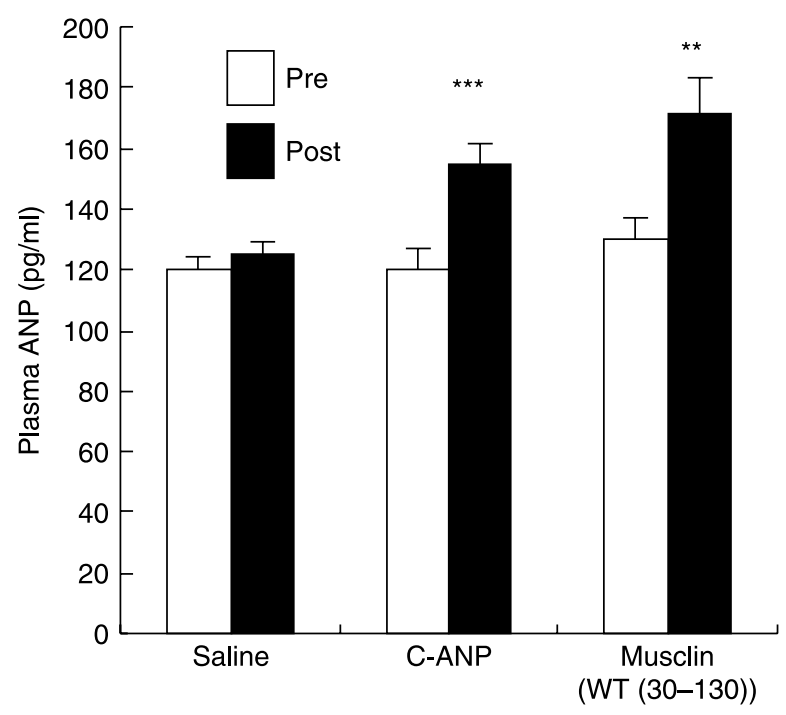

Figure 5 Effects of infused musclin on plasma concentrations of ANP. Plasma ANP concentrations were measured in mice before (pre) and after (post) constant infusion of saline, C-ANP or recombinant full-length musclin (WT (30-130)) for $1 \mathrm{~h}$, as described in Experimental Procedures. Results are mean \pm s.E.M. $(n=8) .{ }^{* *} P<0 \cdot 01,{ }^{* * *} P<0 \cdot 001$ compared with pre-values.

caused a 100-fold reduction in the inhibitory potency compared with full-length musclin. By contrast, the amino acid change of $118 \mathrm{DG}$ in the second ANP-homologous region (Mutant-2) resulted in less than a 10-fold reduction in inhibitory activity. These results provide further evidence for the importance of the first ANP-homologous region of musclin in NPR3 receptor recognition. Additionally, the same amino acid mutations in the cWT mutant produced the same observations. Finally, both mutations in ANP homologous regions (Mutant-3) almost abolished inhibitory activity. The same amino acid mutations in cWT mutant led to similar result. These results suggest that the two regions act cooperatively to achieve the high affinity binding to NPR3.

During the course of this work, high affinity binding of musclin/osteocrin to NPR3 overexpressed in HEK293 cells was reported (Moffatt et al. 2007). The present study further demonstrates that such binding occurs in different cell lines and also by using purified IgG-Fc-NPR3. Although the previous work (Moffatt et al. 2007) concluded that the second ANP-homologous region was more important for binding to NPR3, we show here that the first ANP-homologous region is more significantly involved in the binding to NPR3, based on two independent approaches using four peptides encompassing distinct regions, as well as site-directed mutagenesis in full-length and cWT. We also show that the first and second NP-homologous regions cooperatively play a role in high affinity binding of musclin to NPR3.

We reported previously that musclin gene expression is transcriptionally regulated by nutritional change (Thomas et al. 2003, Nishizawa et al. 2004), and that musclin represses glucose uptake and glycogen synthesis in skeletal muscle cells (Nishizawa et al. 2004). We also identified that musclin gene expression in skeletal muscle could be regulated by FOXO1, a key control switch between anabolism and catabolism in skeletal muscle (Thomas et al. 2003). However, other studies suggested that NPR3 gene expression is decreased markedly by fasting (Sarzani et al. 1995), and therefore, the tissue bioactivity of ANP might be promoted in fasting conditions. Increased ANP bioactivity related to NPR3 expression could be related to increased natriuresis, diuresis, hypotension, and lipolysis under low-calorific diets or fasting conditions (Sigler 1975, Sarzani et al. 1995, Dessì-Fulgheri et al. 1999, Sengenes et al. 2002). ANP also has the switching role in glycolysis from anaerobic to aerobic metabolism (Birkenfeld et al. 2005). NPR3 is expressed in various tissues including adipose, heart, lung, kidney, adrenal, and skeletal muscle (Sarzani et al. 1995, and unpublished data). The previous work (Moffatt et al. 2007), by using osteoblastlineage specific transgenic mouse model, demonstrated that osteocrin/musclin augmented NPs function locally in bone by blocking the clearance action of NPR3. Although this transgenic mouse approach will not totally represent physiological tissue distributions, it is quite interesting that the phenotype of this mouse is similar to the NPR3 knockout mice in the aspect of bone overgrowth, presumably due to the blockage of NPR3. The present study also suggests a possible role for musclin in regulating blood ANP concentrations. Although we have not yet successfully demonstrated the presence of mature peptide or processing fragments of musclin in plasma, the increased and decreased tendency of systolic blood pressure in musclin transgenic mice and null mice respectively, are observed in our preliminary experiments (data not shown). Although the detailed phenotype analysis of these genetically modified mice are needed, we observed the tendency of decreased glucose tolerance by infusing the recombinant musclin in normal C57BL/6J mice, in our preliminary experiments (data not shown). We assume therefore that musclin could play both a paracrine role in regulating the local bioactivity of ANP, by binding to NPR3 expressed in skeletal muscle, and possibly an endocrine role in regulating the systemic availability of ANP for other organs. The exact physiological significance of musclin in the regulation of systemic and local ANP bioactivities is now under investigation.

In conclusion, the present study demonstrated that musclin binds to NPR3 with high affinity and specificity via two intermolecular ANP-homologous regions acting cooperatively. In addition, musclin may regulate the bioactivity of ANP locally and/or systemically.

\section{Declaration of interest}

There is no conflict of interest for all the authors to the study. 


\section{Funding}

This work was supported by the Research Fellowship of the Japan Society for the promotion of Science for Young Scientists no. 9340, Takeda Science Foundation, Senri Life Science Foundation, and The Mochida Memorial Foundation for Medical and Pharmaceutical Research.

\section{Acknowledgements}

We thank Dr Ichiro Kishimoto (National Cardiovascular Center, Suita, Japan) for technical advice on plasma ANP measurement, and Dr Atsunori Fukuhara for helpful comments and discussions.

\section{References}

Almeida FA, Suzuki M, Scarborough RM, Lewicki JA \& Maack T 1989 Clearance function of type $\mathrm{C}$ receptors of atrial natriuretic factor in rats. American Journal of Physiology 256 R469-R475.

Bennett BD, Bennett GL, Vitangcol RV, Jewett JR, Burnier J, Henzel W \& Lowe DG 1991 Extracellular domain-IgG fusion proteins for three human natriuretic peptide receptors. Journal of Biological Chemistry 266 23060-23067.

Birkenfeld AL, Boschmann M, Moro C, Adams F, Heusser K, Franke G, Berlan M, Luft FC, Lafontan M \& Jordan J 2005 Lipid mobilization with physiological atrial natriuretic peptide concentrations in humans. Journal of Clinical Endocrinology and Metabolism 90 3622-3628.

Dessì-Fulgheri P, Sarzani R, Serenelli M, Tamburrini P, Spagnolo D, Giantomassi L, Espinosa E \& Rappelli A 1999 Low calorie diet enhances renal, hemodynamic, and humoral effects of exogenous atrial natriuretic peptide in obese hypertensives. Hypertension 33 658-662.

Maack T, Suzuki M, Almeida FA, Nussenzveig D, Scarborough RM, McEnroe GA \& Lewicki JA 1987 Physiological role of silent receptors of atrial natriuretic factor. Science 238 675-678.

Moffatt P, Thomas G, Sellin K, Bessette MC, Lafrenière F, Akhouayri $\mathrm{O}$, St-Arnaud R \& Lanctôt C 2007 Osteocrin is a specific ligand of the natriuretic peptide clearance receptor that modulates bone growth. Journal of Biological Chemistry 282 36454-36462.
Musarò A, McCullagh K, Paul A, Houghton L, Dobrowolny G, Molinaro M, Barton ER, Sweeney HL \& Rosenthal N 2001 Localized Igf-1 transgene expression sustains hypertrophy and regeneration in senescent skeletal muscle. Nature Genetics 27 195-200.

Nishizawa H, Matsuda M, Yamada Y, Kawai K, Suzuki E, Makishima M, Kitamura T \& Shimomura I 2004 Musclin, a novel skeletal muscle-derived secretory factor. Journal of Biological Chemistry 279 19391-19395.

Potter LR, Abbey-Hosch S \& Dickey DM 2006 Natriuretic peptides, their receptors, and cyclic guanosine monophosphate-dependent signaling functions. Endocrine Reviews 27 47-72.

Salih DAM, Tripathi G, Holding C, Szestak TAM, Gonzalez MI, Carter EJ, Cobb LJ, Eisemann JE \& Pell JM 2004 Insulin-like growth factor-binding protein 5 (Igfbp5) compromises survival, growth, muscle development, and fertility in mice. PNAS 101 4314-4319.

Sarzani R, Paci VM, Zingaretti CM, Pierleoni C, Cinti S, Cola G, Rappelli A \& Dessi-Fulgheri P 1995 Fasting inhibits natriuretic peptides clearance receptor expression in rat adipose tissue. Journal of Hypertension $\mathbf{1 3}$ 1241-1246.

Sell H, Dietze-Schroeder D \& Eckel J 2006 The adipocyte-myocyte axis in insulin resistance. Trends in Endocrinology and Metabolism 17 416-422.

Sengenes C, Stich V, Berlan M, Hejnova J, Lafontan M, Pariskova Z \& Galitzky J 2002 Increased lipolysis in adipose tissue and lipid mobilization to natriuretic peptides during low-calorie diet in obese women. International Journal of Obesity and Related Metabolic Disorders 26 24-32.

Sigler MH 1975 The mechanism of the natriuresis of fasting. Journal of Clinical Investigation 55 377-387.

Thomas G, Moffatt P, Salois P, Gaumond MH, Gingras R, Godin E, Miao D, Goltzman D \& Lanctôt C 2003 Osteocrin, a novel bone-specific secreted protein that modulates the osteoblast phenotype. Journal of Biological Chemistry 278 50563-50571.

Yasui A, Nishizawa H, Okuno Y, Morita K, Kobayashi H, Kawai K, Matsuda M, Kishida K, Kihara S, Kamei Y et al. 2007 Foxo1 represses expression of musclin, a skeletal muscle-derived secretory factor. Biochemical and Biophysical Research Communications 364 358-365.

Received in final form 12 February 2009

Accepted 20 February 2009

Made available online as an Accepted Preprint 20 February 2009 